\title{
Die Veranstaltung
}

\section{Von Adolf Jens Koemeda}

Emanuel Leopold Peterhans, von den meisten Freunden Manu genannt, knipste den Fernseher aus, gähnte und streckte die Arme über seinem Kopf. Dann stand er auf - sehr langsam, das rechte Knie schmerzte - und ging zu seiner Hausbar. Er genehmigte sich einen Drink, Cynar mit Wasser und Eis.

Nein, er sass normalerweise nicht viel vor dem TV. Nur bei den Sportsendungen konnte er sich oft nicht beherrschen - obwohl er wusste, dass er die Quittung prompt bekommen würde: ein schlechtes Gewissen und ausserdem schlief er schlecht, wenn er lange ferngesehen hatte. Die Olympiade allerdings, fand er, müsse man anders bewerten, die sei, bitteschön, eine grosse Ausnahme. Da war er auch weniger streng zu sich, blieb hie und da mehr als drei Stunden vor dem Bildschirm, und es kam vor, dass er abends, bei der Zusammenfassung der wichtigsten Ereignisse des olympischen Tages, die zweite Weinflasche entkorkte; aber bitte: Hier handelte es sich eher um eine Ausnahme.

Als Peking vorbei war, folgten zwei unschöne Wochen. Seine Stimmung war düster, die Zukunftsphantasien auch, er machte sich viele Sorgen um seine Gesundheit; und er hatte keine Kraft mehr - weder in den Armen noch im Unterleib. Damit hatte er allerdings gerechnet, denn er verfügte ja über ausreichend Erfahrungen: Vor zwei Jahren, nach dem Ende der Fussball-Europameisterschaften, war es nicht anders; schon damals aber, als die Phase des Selbstmitleids und der lähmenden Langweile einsetzte, konnte er sich verhältnismässig rasch aufraffen - er hasste doch diese Zeit der miesen Laune und des sinnlosen Grübelns wie die Pest. Plötzlich, ganz ohne Wecker, stand er eines Tages schon um sechs Uhr auf, zog seine Trainingshose an und ging eine halbe Stunde joggen. Um sieben verliess er, frisch geduscht und rasiert, die Wohnung, und zwar beschwingt und leise pfeifend. Er holte sein Fahrrad aus dem Keller und fuhr - trotz des milden Regens zur Arbeit. Er war sicher kein Weichei!

«Weichei», «Weichei»! Valerie wählte oft diesen beleidigenden Ausdruck, da er aber Streitigkeiten und laute Worte nicht mochte, zog er sich - ja, verletzt und missverstanden fühlte er sich schon - lieber zurück. Er lächelte ... und schwieg. Seine stille, ruhige und eher nachdenkliche Art hatte Valerie allerdings im Laufe der letzten zwei Ehejahre zunehmend gereizt und wütend gemacht; sie reklamierte zuerst laut, später leiser und resignierter und dann wiederum sehr heftig. Das hässliche Wort «Weichei» konnte sie selten zurückhalten, obwohl, das wusste sie genau, gerade dieser Ausdruck kränkte ihren Mann masslos.

»Weichei»! Manu schüttelte den Kopf und ging in die Küche; ein paar Sekunden lang stand er unentschlossen vor dem Kühlschrank: Diesmal blieb er stark! Er kehrte zurück zum Fernseher, schaltete ihn aber nicht ein. Er setzte sich in seinen bequemen Sessel und dachte nach.

Ja, er konnte sich beherrschen; und sich schützen - in Grenzen, natürlich. Er musste sich allerdings schützen, denn die Stimmung zuhause wurde immer gereizter, die Auseinandersetzungen immer heftiger und die Kritiken an ihm immer radikaler und verletzender. Widersprechen, zurückschreien? Nein, das lohnte sich nicht, darüber war er sich im Klaren, das würde nur zu neuen Heftigkeiten führen.

\section{Und was jetzt?}

Das fragte er sich damals oft - vor allem nachts, wenn er nicht einschlafen konnte. Und wenn es ihm endlich doch gelang, musste er nach zwei, drei Stunden wieder aufstehen aufs WC; die Prostatavergrösserung. Nichts Schlimmes, kein Karzinom, ein absolut benigner Prozess, aber immerhin ein Prozess, der ihn oft gebieterisch zu einem WC-Gang zwang und ihm anschliessend die nächste Schlafstörung bescherte.

Dass er etwas unternehmen musste, war ihm klar. Sein Vetter, Conny Peterhans, Apotheker seines Zeichens, hatte ihm schon am Telefon seine Vorstellung klar mitgeteilt und ein paar Tage später bei ihrer traditionellen Sommerwanderung deutlich wiederholt: Vorwärts schauen, Manu! Und handeln. Nicht resignieren! Ihr beide gehört schon seit langem in eine Paartherapie!

War auch seine Meinung. Absolut! Entsprechende Vorschläge hatte er Valerie doch schon x-mal unterbreitet und - nichts! Offen- sichtlich kein Bedürfnis bei ihr nach einer Klärung, Veränderung oder nach einem erfahrenen psychologischen Beistand.

Also! In dem Fall bitte nicht im gleichen alten Geleise weiterfahren. Eine neue Kursrichtung sei fällig, dachte er. Dann könnte man auch von einem Neuanfang sprechen und der Beziehung weiterführende Impulse geben. Einen «tollen Schub an innovativer Energie» formulierte er im Stillen und sagte es sogar laut - das Ganze klang dann noch wuchtiger und überzeugender. Wer sollte aber anfangen, fragte er sich, von wem müsste die Initialzündung wohl kommen? Ihm war klar: Den ersten Energieschub konnte nur er liefern. Er nickte, schmunzelte ein wenig und in dem Augenblick kam ihm Ramuz in den Sinn: «Schönheit entsteht durch Endlichkeit; jeder Neubeginn braucht auch ein Ende.» Toll! Er entschloss sich, diesen Spruch in seine Zitatensammlung aufzunehmen.

Die Laune besserte sich langsam. Manu erhob sich, ging in die Küche zum Kühlschrank und holte sich eine Flasche Weisswein. Fendant. Ein Glas stand schon auf dem TV-Tischchen, ein hübsches Ding aus der Westtürkei, vor zwei Jahren bei der letzten noch gemeinsamen Ferienreise gekauft. Er schenkte sich ein.

Als erstes: Weg von hier! Er dachte an die Firma, an die Wertschätzung, die er dort genoss - seit Jahren! Deshalb durfte er doch annehmen, dass er bei seinen Vorgesetzten Verständnis für seinen Wunsch finden würde: Bitte nicht weiter im Büro sitzen müssen, lieber hinaus aus der Bude, lieber in den Aussendienst.

Er hatte sich, Gott sei Dank, nicht getäuscht. Es war nicht einfach, im Prinzip aber machbar. Allerdings nicht sofort. Bei einer Besprechung erfuhr er, zumindest eine betriebsinterne Umschulung sei notwendig, vier Wochen; das gelte für alle, hier dürfe man keine Ausnahmen machen. Man bat ihn um Verständnis.

Und es klappte! Schon zwei Monate nach der klärenden Besprechung bei der PharmaDirektion fing er mit der Umschulung an und vier Wochen später läutete er zum ersten Mal an der Tür eines Allgemeinpraktikers. Sein Terminkalender füllte sich schnell. Mittwochs 
kam er ab und $\mathrm{zu}$ nachhause, eher selten, meistens erst am Wochenende.

Die Stimmung daheim besserte sich allmählich, es trat also ein, womit er gerechnet hatte - Entspannung. Was er aber am Anfang nicht wusste: Nicht die Länge der berufsbedingten Abwesenheit war der Grund dafür, sondern ein Freund. Ja, ein nigelnagelneuer Freund seiner Frau: zwei Jahre jünger als sie, auch Akademiker und Chemiker wie er, erst seit kurzem geschieden und - das nahm er halt an - kein «Weichei».

Manu stand auf und schüttelte den Kopf.

Ja, ja, ein «Weichei», ein «Langweiler», und leider auch die derbe Variante: «Du hast einfach keinen Pfeffer im Arsch.» All das waren Ausdrücke seiner Frau, die er sich in der finalen Phase ihrer achtjährigen Ehe fast täglich hatte anhören müssen. Genug! Er war nicht mehr bereit, sich dieses verzerrte und beleidigende Bild überstülpen zu lassen! Einverstanden: ständig auf Achse oder gar ein rastloser Draufgänger war er nie gewesen - aber ein «Weichei»? Das bitte auch nicht! Und zwar nicht nur in seinen eigenen Augen, sondern - das war für ihn sehr wichtig - ebenfalls in den Augen von vielen gemeinsamen Bekannten und Freunden.

Genug für heute! Er brachte sein Glas in die Küche und spülte es; und er liess es auf dem Küchentisch stehen. Für alle Fälle.

Er sah es so: Seine aktive Seite war immer vorhanden gewesen, wenn auch zeitweise neben Valeries Angetriebenheit und wuchtigen Dauerpräsenz - ziemlich verdrängt und im Laufe der Jahre, leider, ein wenig verkümmert. Erst nach ihrem Auseinandergehen auf dem hatte er bestanden, schon zwei Wochen nach dem Bekanntwerden der Untreue waren sie ein getrenntes Paar - ja, erst dann meldete sich seine alte Kraft wieder. Zum Beispiel: Weder zog er die Decke über den Kopf noch blieb er tagelang der Arbeit fern. Er gestaltete jetzt seine Freizeit überlegt und einfallsreich, und zwar nicht nur an Wochenenden, sondern auch unter der Woche.

Valerie verhielt sich, was das Materielle anbelangte, weitgehend fair: Die Schuld am Scheitern der Ehe lag eindeutig bei ihr, und sie war nun bereit, die Konsequenzen zu tragen; auch die finanziellen. Sie erwartete keine monatlichen Zuwendungen von ihm, und was die gemeinsame Immobilie betraf - ein schönes Ferienhaus im Toggenburg -, zeigte sie sich sympathisch grosszügig (dass ihr neuer Freund recht vermögend war, wusste Manu damals noch nicht). Er konnte es sich deshalb leisten, seinen Firmeneinsatz zu reduzieren, was konkret bedeutete: Herabset- zung der Arbeitsverpflichtung von hundert auf fünfundsiebzig Prozent.

Ja, das tat er. Schon zwei Monate nach der Trennung hatte er einen neuen Vertrag in der Tasche. War das aber richtig? fragte er sich eines Tages. Nun, darüber war er sich nicht im Klaren. Er hatte sich einmal so entschieden, und jetzt wollte er an diesem Entschluss nicht mehr rütteln. Man könne doch, so tröstete er sich, auch beim Umgang mit der Freizeit Erfahrungen sammeln und später sogar ein guter Routinier werden.

An eine neue Bindung dachte Manu noch nicht, dafür war die Überraschung, ja der Schock der Trennung zu gross gewesen. Er wusste allerdings: Seinen Freundeskreis konnte man zwar nicht als riesig bezeichnen, dafür aber waren seine Interessen ziemlich zahlreich und breit gefächert, und gerade das schien ihm in dieser schwierigen Zeit enorm wichtig zu sein.

Fussballübertragungen, zum Beispiel, hatte er sich schon früher angeschaut; mehr Ausdauer und Engagement hatte er erst nach den letzten Europameisterschaften aufgebracht. Bei seinen beliebten Sportarten rangierte auch Tennis ganz oben; bei den letzten US-Open hatte er ab dem Viertelfinale kein einziges Match mehr versäumt. Und dennoch: Er war sonst kein typischer Fernsehhocker! Motorsport, Leichtathletik und die gröberen Sportarten - wie Ringen und Kickboxing - interessierten ihn überhaupt nicht.

Keine Kinder, seit einem guten Jahr die Frau weg, das Arbeitspensum reduziert - über einen Mangel an Freizeit konnte er sich wirklich nicht beklagen; und dass die optimale Einteilung der Stunden weder dem Zufall noch seiner momentanen Laune überlassen werden durfte, das wusste er auch. Er plante deshalb seine verschiedenen Unternehmungen langfristig, das heisst, er berücksichtigte bewährte, sich wiederholende Veranstaltungen, und darüber hinaus strukturierte er jede Woche neu. Er ging dabei von guten Zeitungskritiken, interessanten Zusatzangeboten und aktuellen lokalen Überraschungen aus. Sport, Musik, Theater, Kultur allgemein, ja, alle Richtungen sollten vertreten sein, nichts Wichtiges durfte zu kurz kommen.

Manu setzte sich endlich in seinen Fernsehsessel und liess die raffiniert eingebaute Fussstütze mit einem präzisen Kick ausfahren. Weiter aber nichts. Der grosse Flachbildschirm blieb stumm.

Die Olympiade, sinnierte er, ja, das war doch ein toller Höhepunkt gleich zu Beginn des Sommers gewesen. Er hatte sich nicht nur die aufwendige Eröffnungszeremonie angeschaut, sondern auch die meisten Sendungen über die spannende Zeit des Stadionbaus ... er löste nun den engen Ledergürtel und machte den obersten Knopf am Hosenbund auf. Dann legte er seine Hände in den Schoss; seit drei, vier Wochen spannte alles um die Taille, er fand es unschön und ärgerte sich darüber. Dass er in der letzten Zeit ein paar Kilo zugenommen hatte, ahnte er, wie viele genau, nein, das war ihm unbekannt; eine systematische Gewichtskontrolle unterliess er absichtlich, denn er wusste, eine genaue Gewichtsprüfung würde ihn nur deprimieren.

Heute kein Fendant mehr. Er schenkte sich Whisky ein, ein kleines Gläschen. Er roch daran, schnalzte mit der Zunge und trank. Beinahe wäre ihm ein leiser Rülpser entwichen, er unterdrückte ihn jedoch im letzten Moment.

Die Olympiade! Die Nostalgie packte ihn wieder. Die Spiele, das Fest der Sommerfeste, könnte, wenn es nach ihm ginge, einmal in zwei Jahren stattfinden. Und die US-Open oder Wimbledon? Wenn er entscheiden dürfte - zwei Mal im Jahr.

Aber wie gesagt - wegen des grossen TVBildschirmes zuhause, die Diagonale weit über einen Meter zehn, vernachlässigte er die wichtigsten Veranstaltungen keineswegs.

Die Festmeile in Kreuzlingen, zum Beispiel; in diesem Jahr war er zum ersten Mal dabei gewesen. Guter Jazz, gutes Cabaret, gute Stimmung ... eine noch junge Sache, aber eine Veranstaltung mit Zukunft, da war er sich sicher; er würde im nächsten Jahr wieder gehen, vielleicht auch ein paar Freunde mitnehmen. Und bald danach die Olma. Dieser Event in St. Gallen war wichtig für ihn; schon seit Jahren fuhr er hin, zu Beginn mit Valerie, später alleine. Besonders deutlich erinnerte er sich an die Düfte: an original St. Galler Schüblig, zum Beispiel, mit scharfem Senf und dunklem Brot.

Manu stand auf, ging in die Küche und öffnete den Kühlschrank. Na ja! Auf Anhieb fand er nichts, wonach es ihn wirklich gelüstete; er gab sich also mit Aoste Kochschinken zufrieden und nahm Apfel-Kren-Sosse dazu. Er kaute langsam und gründlich und setzte sich dann wieder in seinen Sessel; er betrachtete die graue Scheibe des ausgeschalteten Fernsehers, in dem sich eine moderne Stehlampe im Fenster spiegelte. Warum, fragte er sich, warum reiste er gerade in die Ostschweiz - und warum so oft?

Nun, diese Gegend zog ihn an. Schon immer. Eigenartig! Die Eltern kamen aus dem 
Aargau, und er selber war in der Nähe von Zürich aufgewachsen. Aber nicht nur die Ostschweiz, für Feste, Ausstellungen und Kultur überhaupt fand er auch Österreich grosse Klasse. Im Zumtor-Museum in Bregenz, zum Beispiel, hatte er in der letzten Zeit kaum eine Vernissage verpasst, das Haus faszinierte ihn. Und ebenfalls bei den Festspielen am See war er schon seit Jahren zu Gast, am Anfang mit, zuletzt ohne Valerie. Ja, Bregenz! Wenn ich an die tollen ersten Wochen dieses Sommers zurückdenke, sagte er sich, bekomme ich nur Heimweh - und nasse Augen - apropos Augen: die Toska!

Er erinnerte sich nun an das grossartige Bühnenbild, an das Auge vor allem: ein riesiges Auge! Schon nur die Pupille gut drei Meter gross. Viele Bilder tauchten jetzt auf, verschiedene Farben, nicht bloss das dunkle Grün der Iris, obwohl, ja, die faszinierte ihn besonders. Er hatte damals immer wieder hinschauen müssen und dabei die Schauspielerei stellenweise vergessen - die Pupille war ihm viel wichtiger gewesen. Er guckte sie an, Aug in Auge sozusagen - lange; da sei, dachte er, schon fast eine hypnotische Wechselwirkung im Spiel gewesen.

Tja, wie er plötzlich nach Locarno kam, war ihm unklar und für ihn aber im Augenblick auch nicht wichtig. Er war doch oft in Locarno gewesen, beim Filmfestival, nur dann eigentlich - ob er wirklich jedes Jahr hingefahren sei, hm, das wusste er nicht mehr genau; er könnte allerdings in seine Agenda schauen - wenn es jemanden interessieren würde.

Schade, die Menschen interessierten sich immer weniger für Kultur; er bedauerte das und goss sich ein zweites Glas Whisky ein. Jawohl, das grosse Auge auf der riesigen Wasserbühne in Bregenz hatte ihm wirklich gefallen, es imponierte ihm sehr, es hatte Charisma. Nun, das hätte er eigentlich auch gerne, viel Charisma, viel mehr, als er tatsächlich besass, ein riesiges Charismavolumen bräuchte er, wie Obama - nein nein, mit dem Amerikaner wollte er sich jetzt nicht vergleichen - obwohl, gar nicht so unwichtig, das optimale Charismapotential. Hätte er mehr davon gehabt, hätte ihn Valerie, wahrscheinlich, nicht immer so gedemütigt, missachtet oder mit tagelangem Schweigen und Wutausbrüchen bestraft. Und sich zuletzt in einen anderen Mann verliebt!

Oh ja, viel hatte er im Laufe der Jahre geschluckt, schlucken müssen, zu viel, vielleicht hätte er das nicht so lange zulassen dürfen. Jetzt machte er sich Vorwürfe, wobei - am liebsten hätte er sich geohrfeigt, gut, es könnte auch etwas Masochistisches dahinter stecken; sogar die einschlägigen Fantasien waren ihm nicht ganz fremd - und auch die entsprechenden Handlungen nicht: Er ging, zum Beispiel, bei kalter Witterung immer sehr gerne ins Wasser; im Herbst bei Lufttemperaturen von 15 oder 16 Grad, niemand verstand das, alle schüttelten nur den Kopf.

Auch in Bregenz war es so gewesen. Ein sehr kalter Abend und trotzdem war er ins Wasser gegangen - nur so, nackt. Er stieg in den See und schob die Bühneninsel mit dem riesigen Auge in Richtung Konstanz. Sie kam eigenartigerweise gleich wieder zurück, als ob ein Gummiseil sie ans Ufer zöge, er musste also noch einmal ins Wasser. Wieder stiess er die Augeninsel weg, westwärts, aber diesmal war sie viel schwerer als vorher; sie blieb stehen, als ob jemand einen Anker geworfen hätte. Er zerrte lange an den Seilen und war danach nicht nur müde, sondern richtig erschöpft. Peinlich! Etwa vierhundert Menschen hatten ihm dabei zugesehen! Tja! Und sonst, wenn er schon bei den Zahlen war: hundertsiebzigtausend Besucher pro Spielzeit - hatte er irgendwo gelesen.

Das Filmprogramm des Festivals hatte er aus dem Internet und hatte sich sofort entschieden. Per Mail eine Karte bestellt. Und eine Bestätigung erhalten. Sorglos nach Locarno gereist, diesmal mit dem Auto. Und dann die Überraschung: Auf seinen Namen war keine Karte reserviert; und seit zwei Tagen ausverkauft - absolut hoffnungslos.
Geschimpft, ja, das hatte er, war aber anständig geblieben; hatte keine Kraftausdrücke benützt und auch keine Ausuferung zugelassen, sonst hätte er sich vor den Herumstehenden nur lächerlich gemacht. Moment! Also doch ein «Weichei»? Ach! Nicht die Spur! Er marschierte sofort zur Polizei - merkwürdig! Er landete bei der Wasserpolizei; einen anderen Posten, sagte man ihm, gebe es nicht mehr, schon vor mehreren Jahren abgeschafft. Lediglich die Wasserpolizei gebe es hier. $\mathrm{Na}$ so was!

In diesem Augenblick kam ein kurzer und sehr eruptiver Schnarcher. Er war allerdings nicht mächtig genug, um Manu zu wecken. Nur die auf dem Schoss zusammengefalteten Hände lösten sich und rutschten auf den beigen Veloursstoff der Sitzfläche; seine Traum-Reise ins Tessin unterbrach er deshalb noch lange nicht. Hundertsiebzig Besucher pro Spielzeit, flüsterte er und kratzte sich am Oberschenkel und zwischen den Beinen. Wo aber? Hier in Bregenz oder doch in Locarno, beim Filmfestival? Das wusste er nicht. Er bedauerte es, seufzte, kratzte sich noch heftiger - und erwachte.

Tja! Ein Nachmittagsschlaf. Ein kurzer Schlummer, aber ein Schlummer, genau das, was er immer zu vermeiden bemüht war. Warum? Weil er die düstere Laune, mit der er jedes Mal erwachte, nicht mochte. Er seufzte noch lauter, denn er wusste, jetzt konnte er das Sofa kaum wieder verlassen, und wenn es ihm später doch gelingen sollte, dann nur,

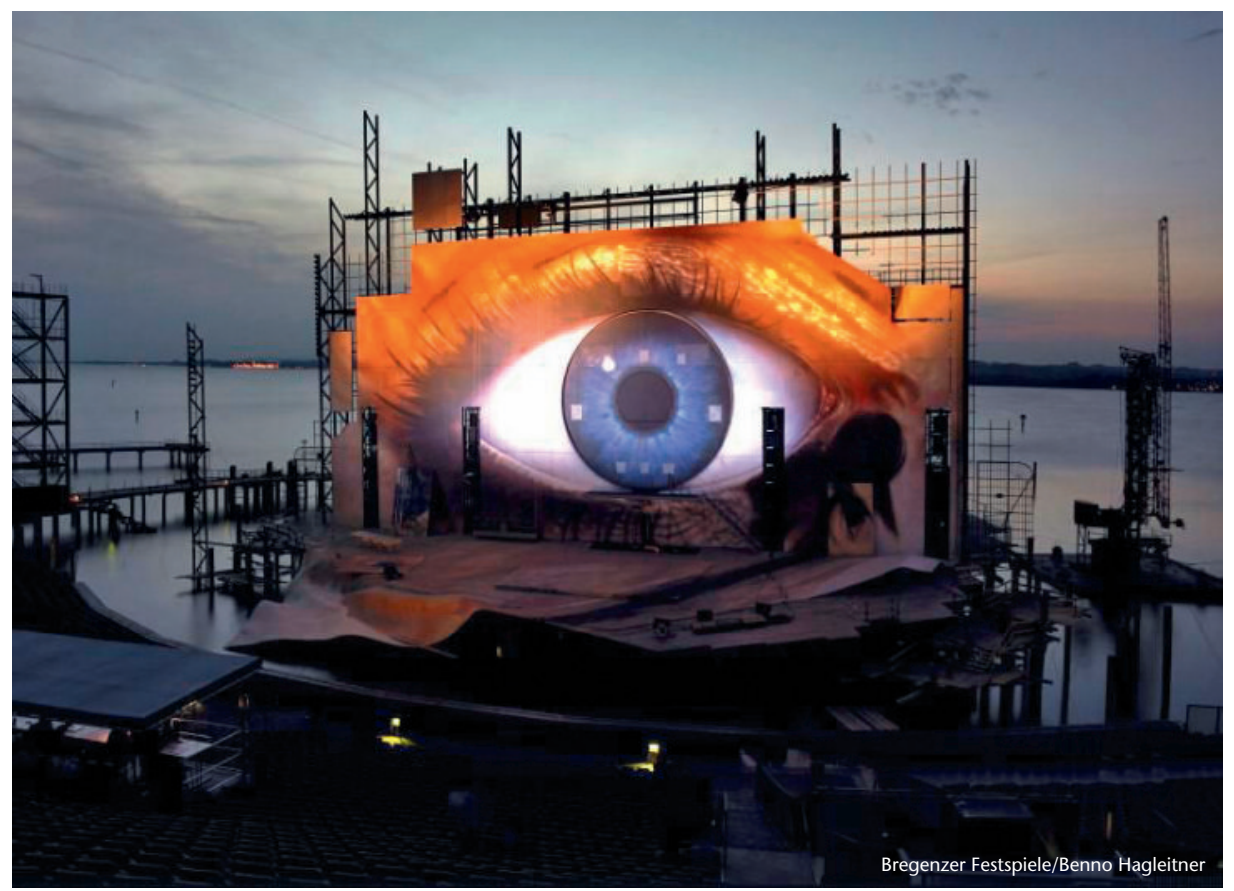


um direkt ins Schlafzimmer zu gehen, ins Bett.

Locarno oder Bregenz? Nun, diese Frage beschäftigte ihn jetzt nicht mehr; eine andere - neue - Idee meldete sich: Bald werde der Herbst kommen. Er mochte diese Zeit nicht! Die Blätter würden zwar schön gelb werden, ja, schön, das war aber auch schon alles. Was dann noch folgen würde, konnte seine Stimmung keineswegs heben: Kälte, oft Regen, immer kürzer werdende Tage, Dunkelheit.

Nun, ein kleines Licht am Horizont war, Gott sei Dank, sichtbar. Beim Oktoberfest machte er jedes Mal mit, natürlich, im letzten Jahr allein. Und das Neue: Nicht bis nach München würde er nun fahren müssen, denn das Fest gab es jetzt auch in Schaffhausen. Nicht das Gleiche, gewiss, aber ordentlich, durchaus vergleichbar. Er war sehr froh über dieses unerwartete Zusatzangebot und überhaupt: Er musste doch zufrieden sein, dass er eine gute Stelle hatte, gesund war und noch lange nicht zum alten Eisen gehörte.

Das Tief des Nachmittagsschlafs war allmählich überwunden, er summte jetzt eine bekannte Beatles-Melodie; dann stand er auf und wollte eine CD auflegen. Vor dem Player machte er allerdings halt, es zog ihn zum anderen Gerät: Er setzte sich an seinen Arbeitstisch, schaltete den PC ein und ging ins Internet. Die Vorstellung, bis Mitte Oktober keine bedeutsame Veranstaltung ausfindig zu machen, fand er plötzlich ungemütlich, unfair und, ja, das auch, bedrückend. Aber leider - es war so. Enttäuscht liess er seinen PC runterfahren.

Was den August betraf, gewiss, da musste er sich keine ernsthaften Sorgen machen. Das neue Heidi-Musical in Walenstadt beschloss er, ersatzlos zu streichen. Dafür würde er wahrscheinlich im August nach Olten reisen. «Silo 8», ein spektakulärer Openair-Streich; es klang interessant: Die Gegenwart und die Zukunft gehören nur der Jugend, dann aber brechen die «Alten» auf und bringen «das ganze System» zum Einsturz ... na ja! Er konnte sich zwar - alles auf der Bühne! - wenig darunter vorstellen, umso grösser waren aber sein Interesse und seine Neugierde.

Nein, um den August musste er keine Angst haben, an Veranstaltungen mangelte es keineswegs; und es gab sogar eine gewisse Steigerung. Zum Beispiel Ende August - ein Römerfest. Für ihn ganz klar, wieder ein Muss; das hatte er sofort gewusst, gleich nach dem flüchtigen Blick auf den Flyer «Römerstadt Augusta Raurica» in Augst bei Basel. Alles dabei: römische Werkstätten, römische Mahlzeiten im Liegen auf dem weissen römischen Bett und, ja, das bitte auch: heftige Gladiatorenkämpfe. Er war begeistert! Telefonisch hatte er schon vor Tagen weitere Prospekte bestellt und sie an einige seiner Freunde weitergeleitet.

Gar keine Frage: August - o.k. Eher September war das grosse Problem. Im Augenblick wusste er noch nichts, war aber überzeugt, dass sich mit der Zeit schon etwas Vernünftiges zeigen würde. September war ja keine schlechte Jahreszeit - die Sommerferien gerade vorbei und das Wetter meist etwas stabiler und wärmer als im Frühling.

Römer-Weekend 25./26. August. Er blätterte im Terminkalender ... bei diesem Datum keine Notiz. Möglicherweise das Wochenende danach? Römerfest-Schlusstag? Wahrscheinlich hatte er die Eintragung vergessen. Er stand auf, suchte einen Flyer, zwei, drei Stück hatte er sicher behalten. Wo waren sie aber? Keine Ahnung! Hatte er doch alle weggeschickt? Die unangenehme Suche im Stoss der alten Zeitungen wollte er sich ersparen, er ging lieber noch einmal ins Internet. Er war allerdings unkonzentriert und mit der Zeit sogar gereizt; die Suche dauerte länger als sonst. Dann sagte er sich, dass das Einfachste doch meist das Beste sei, und er tippte: www. römerfest.ch

Voilà! Das richtige Bild war sofort da. Mit ihm aber auch die grosse Überraschung: Nichts! Werde diesmal, leider, ausfallen müssen; die Gemeindeverwaltung ... technische Gründe ... die ...

Manu las nicht mehr weiter. Ist das die definitive Entscheidung? fragte er sich. Lässt sich wirklich nichts mehr machen? Oder doch? Vielleicht sollte er zuerst Dr. Martens anrufen, seinen Juristen. Der könnte eventuell - ach nein, das würde wahrscheinlich nicht viel bringen, nur sinnlose Geldausgaben. Erzwingen lässt sich hier kaum etwas, leider.

Er holte nun seinen Terminkalender und blätterte noch einmal darin: Im Oktober, ja ja, da gab es genug - aber im September? Kein Fest, keine bedeutsame Sportveranstaltung, kein Event überhaupt. Und sonst - private Einladungen, Vernissagen? Auch nichts! Die Kalenderseite war jungfräulich leer.

Nichts. Nichts!

Diese zwei Worte sagte er nun halblaut vor sich hin und erschrak. Seine eigene Stimme kam ihm plötzlich kalt, unsympathisch, vor allem aber fremd vor. Er sprach jetzt ein wenig lauter und versuchte, dem Tonfall ein melodisches und warmes Timbre zu geben: kein Fest ... kein Event ... OpenairTheater auch nicht ... überhaupt keine Veranstaltung.

Es misslang. Das Kalte und Fremde blieb. Er ging zu seinem TV-Sessel, schaltete allerdings den Fernseher nicht an. Nach einigen Sekunden stand er wieder auf; er schlurfte ins Badezimmer und blieb vor dem Doppelwaschbecken stehen - ein breites Ding in Granitfassung, montiert in der besten Zeit seiner Ehe. Aber was wollte er hier eigentlich? Ein Glas Wasser trinken? Pinkeln? Ohrenhaare entfernen? Sich rasieren kaum, das hatte er vor gut zwei Stunden bereits gemacht. Er wusste es nicht.

Manu war enttäuscht. Sehr enttäuscht! Nicht nur von Valerie, eher von sich selber. Heute aber auch vom Wetter, von der gähnenden Leere im Kalender auf der Septemberseite und - das vor allem - von den Versagern in Augst.

Kein Event! Er legte sich aufs Bett und flüsterte das «Nichts» noch mehrere Male. Er wünschte sich dabei, dass dieses Wort auch sein letztes Wort sein möge: Nicht nur das des heutigen Tages, sondern überhaupt.

Tja! 